\title{
Clinical and Dosimetric Implications of Air Gaps between Bolus and Skin Surface during Radiation Therapy
}

\author{
Yousaf Khan $^{1}$, J. Eduardo Villarreal-Barajas ${ }^{1}$, Mona Udowicz ${ }^{1}$, Richie Sinha ${ }^{1}$, Wazir Muhammad ${ }^{2}$, \\ Ahmed N. Abbasi ${ }^{3}$, Amjad Hussain ${ }^{3 *}$ \\ ${ }^{1}$ Department of Radiation Oncology, Tom Baker Cancer Centre, Calgary, Canada; ${ }^{2}$ Institute of Nuclear Medicine, Oncology and \\ Radiotherapy, Abbottabad, Pakistan; ${ }^{3}$ Radiation Oncology, Department of Oncology, Aga Khan University Hospital, Karachi, Paki- \\ stan. \\ Email: *amjadso_76@yahoo.com
}

Received July $13^{\text {th }}, 2013$; revised August $15^{\text {th }}, 2013$; accepted August 22 ${ }^{\text {nd }}, 2013$

Copyright (C) 2013 Yousaf Khan et al. This is an open access article distributed under the Creative Commons Attribution License, which permits unrestricted use, distribution, and reproduction in any medium, provided the original work is properly cited.

\begin{abstract}
Purpose: The main objective of the study was to evaluate the effect of air gaps of $0-5.0 \mathrm{~cm}$ between bolus and skin for $1.0 \mathrm{~cm}$ Superflab bolus on surface dose $\left(\mathrm{D}_{\text {Surf }}\right)$ and depth of maximum dose $\left(\mathrm{d}_{\max }\right)$ in solid water and Rando ${ }^{\circledR}$ phantoms. Methods: In this work, the effects of bolus to surface distance on $\mathrm{D}_{\text {Surf }}$ and variation in $\mathrm{d}_{\max }$ were analyzed in a solid water phantom and in an anthropomorphic Rando ${ }^{\circledR}$ phantom for different field sizes, using Gafchromic ${ }^{\circledR}$ EBT films and farmer chamber. Results: For field sizes of $5 \times 5 \mathrm{~cm}^{2}$ the $D_{\text {Surf }}$ is significantly affected by increasing air gaps greater than $5 \mathrm{~mm}$. For field sizes larger than $10 \times 10 \mathrm{~cm}^{2}, \mathrm{D}_{\text {Surf }}$ is nearly the same for air gaps of $0-5.0 \mathrm{~cm}$. For small fields and $6 \mathrm{MV}$ photon beam, $\mathrm{d}_{\max }$ increases with increasing air gap, while for $10 \mathrm{MV}$ beam and smaller field sizes (i.e. $5 \times 5$ and $10 \times 10 \mathrm{~cm}^{2}$ ) the $\mathrm{d}_{\max }$ first decreases and then increases with the air gaps. For both 3DCRT and IMRT plans on Rando $^{\circledR}, D_{\text {Surf }}$ reduction is more prominent with increasing air gaps. Conclusion: For field sizes larger than $10 \times 10 \mathrm{~cm}^{2}$ $\mathrm{D}_{\text {Surf }}$ is largely unaffected by air gaps. However, smaller air gap results in shallower $d_{\max }$ for both $6 \mathrm{MV}$ and $10 \mathrm{MV}$ photon beams at all fields sizes. Special consideration should be taken to reduce air gaps between bolus and skin for field sizes smaller than $10 \times 10 \mathrm{~cm}^{2}$ or when surface contour variations are greater or when the bolus covers small area and at the border of the field.
\end{abstract}

Keywords: Bolus Distance; Skin Dose; IMRT; Dose Build-Up

\section{Introduction}

High energy photon beams typically have a lower dose at skin $\left(D_{\text {Surf }}\right)$ than dose maximum $\left(\mathrm{d}_{\max }\right)$ at depth. This phenomenon is known as "skin sparing" and estimated that $\mathrm{D}_{\text {Surf }}$ can be as low as $25 \%$ of the dose at $\mathrm{d}_{\max }$. For treating near surface tumors, bolus is placed on the surface in order to increase $\mathrm{D}_{\text {Surf. }}$. The effect of dose build-up is more prominent in Mega Voltage (MV) photon beams [1]. $D_{\text {Surf }}$ and $d_{\max }$ depend on photon beam energy, field size, beam modification devices, SSD and angle of incidence. These also depend on electron contamination from the flattening filter, beam modifiers and air [2-4]. Accurate measurement of $\mathrm{D}_{\text {Surf }}$ doses in RT can provide valuable information for clinical use to avoid near surface recurrences while at the same time limiting severe skin toxicity $[5,6]$.

Hsu et al. [7] reported no significant differences in

"Corresponding author.
$\mathrm{D}_{\text {Surf }}$ between IMRT and conventional radiotherapy techniques. Lee et al. [8] found that the average increase of $\mathrm{D}_{\text {Surf }}$ was about $18 \%$ due to bolus effect of thermoplastic shell. They also investigated that with thermoplastic shell $D_{\text {Surf }}$ was $84 \%$ and $100 \%$ of the prescribed dose for parallel opposed (POP) and IMRT treatments respectively. Higgins et al. [9] demonstrated that $\mathrm{D}_{\text {Surf }}$ using POP, tomotherapy and IMRT were $69 \%, 71 \%$ and $82 \%$ respectively. Dogan and Glasgow [10] reported that $\mathrm{D}_{\text {Surf }}$ with $6 \mathrm{MV}$ photon beam IMRT were $8 \%$ and $6 \%$ lower than those of the open field for zero and $75^{\circ}$ gantry angles respectively. Yokoyama et al. [11] stated that $\mathrm{D}_{\text {Surf }}$ with IMRT was $10 \%$ lower than the open field treatment. Gray et al. [12] reported in their investigation of PDD measurements that as the air gap increases from 1 to 15 $\mathrm{cm}$, the dose reduces at the surface. In order to increase $\mathrm{D}_{\text {Surf }}$ in conventional total body irradiation (TBI) an acrylic sheet of $1 \mathrm{~cm}$ thickness is placed in front of the 
patient at 15 to $20 \mathrm{~cm}$ from the skin surface.

In photon beam radiation therapy such as breast and chest wall it is desirable to predict the dose delivered to skin, superficial nodes and/or any remnant in the surgical scar of the patient for better treatment outcome. It is also important to know any injury caused by radiation to the skin. Many biological effects such as skin reactions are correlated with basal cell layer damage. The basal cell layer is located at about $0.07 \mathrm{~mm}$ depth and it is very radio-sensitive.

Main objective of this study was to evaluate $\mathrm{D}_{\text {Surf }}$ using a $1.0 \mathrm{~cm}$ Superflab bolus while introducing various air gaps $(0 \mathrm{~cm}-5.0 \mathrm{~cm})$. The following scenarios were investigated:

1) Is zero-air gap absolutely necessary between skin and bolus during real treatment delivery?

2) How depth of maximum dose $\left(d_{\max }\right)$ is affected by the distance between bolus and skin surface.

As discussed earlier the $\mathrm{D}_{\text {Surf }}$ also depends on the delivery technique in addition to bolus-surface distance (BSD). Therefore doses were also measured and compared for 3D-CRT and IMRT techniques. The goal of these measurements was to demonstrate the impact of the delivery technique on the $D_{\text {Surf }}$ in the presence of air gaps in real clinical situations.

\section{Materials and Methods}

The effects of Superflab (Med-Tec, Orange, IA) bolus to surface distance on the $D_{\text {Surf }}$ and variation in the $d_{\max }$ were analyzed in a solid water phantom (Gammex RMI Model 457, Middleton, WI) and in an anthropomorphic Rando ${ }^{\circledR}$ phantom (The Phantom Laboratory, Salem, NY).

Rando $^{\circledR}$ phantom was used to simulate head and neck Intensity Modulated Radiotherapy (IMRT) and rectum 3D-CRT treatment techniques. All measurements were performed on a $2100 \mathrm{C}$ (Varian, Palo Alto, CA) linear accelerator (6 and $10 \mathrm{MV})$, equipped with 120-leaf Millennium MLC.

An exradin ionization chamber (Model A12) was used for dose measurement in solid water phantom and to provide reference measurements for Gafchromic ${ }^{\circledR}$ EBT (International Specialty Products, NJ, USA) film measurements. Calibration of the Gafchromic ${ }^{\circledR}$ EBT film was performed for a range of doses (5 to $300 \mathrm{cGy}$ ). Dose measurement accuracy of the Gafchromic ${ }^{\mathbb{B}}$ EBT film was better than $\pm 2 \%$ with \pm 1.7 standard deviation. Each batch of the Gafchromic ${ }^{\circledR}$ EBT films was calibrated separately.

\subsection{Dose Measurements in Solid Water Phantom}

Gafchromic ${ }^{\circledR}$ EBT film can potentially be used for $D_{\text {Surf }}$ measurement. It is also considered a useful tool for accurate dose measurement near the surface (i.e., within a depth of a few $\mathrm{mm}$ ), and for CNS junctions. Radiochro- mic films were cut in two different shapes for surface (3 $\left.\times 3 \mathrm{~cm}^{2}\right)$ and depth $\left(11 \times 2 \mathrm{~cm}^{2}\right)$ dose measurements. Depth dose profiles were obtained with films sandwiched vertically in slabs of solid water. For $\mathrm{D}_{\text {Surf }}$ measurements the square films were placed on top of the solid water phantom at beam central axis. The separation between the phantom surface and the bolus was adjusted with Styrofoam sheets of $0.5,1.0,2.0,3.0,4.0$ and $5.0 \mathrm{~cm}$ as shown in Figure 1. Measurements with bolus placed right on top of the water phantom $(0 \mathrm{~cm}$ distance) and without bolus were also performed. The source to phantom surface distance was kept constant at $100 \mathrm{~cm}$.

Doses (PDDs and $\mathrm{D}_{\text {Surf }}$ ) at the central axis with and without bolus material were measured for $5 \times 5 \mathrm{~cm}^{2}, 10$ $\times 10 \mathrm{~cm}^{2}, 15 \times 15 \mathrm{~cm}^{2}$ and $20 \times 20 \mathrm{~cm}^{2}$ fields. All the films were exposed to $100 \mathrm{cGy}$. The Gafchromic ${ }^{\circledR}$ EBT film readings (optical densities) were converted to doses using the calibration curve. All the dose profiles were normalized to the maximum dose obtained with $0 \mathrm{~cm}$ bolus to skin air gap.

\section{2. $D_{\text {Surf }}$ Measurements on Rando ${ }^{\circledR}$ Phantom}

IMRT and 3D-CRT plans were created using Eclipse on a Rando ${ }^{\circledR}$ phantom: one for head and neck and another for a rectum case with a prescribed dose of $200 \mathrm{cGy}$ to the reference point using $6 \mathrm{MV}$ beam. The head and neck IMRT plan was calculated for 5 fields. The rectum plan was delivered with 4-field's box technique.

Both the plans require the $\mathrm{D}_{\text {Surf }}$ to be $95 \%$ of the pre-

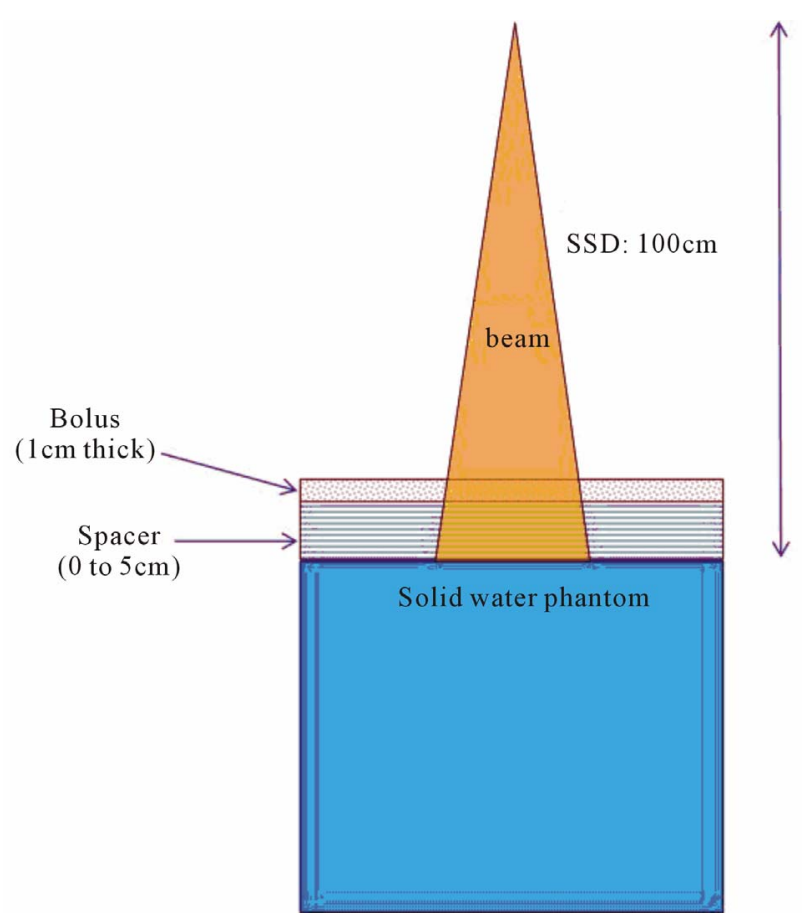

Figure 1. Geometrical setup for surface and depth dose measurements using solid water phantom. 
scribed dose. Custom boluses were made for both plans. Both plans were delivered without bolus and with bolus at various distances from the skin.

The Rando ${ }^{\circledR}$ phantom was aligned using lasers on the treatment couch according to the setup markings. $D_{\text {Surf }}$ were measured for four locations (marked as 1, 2, 3 and 4 on the films) for the head and neck case and one position for the rectum case as shown in Figure 2. Four strips were placed at position 1, 2, 3, and 4 across the IMRT fields from inferior to superior direction.

\section{Results}

To choose a suitable bolus for optimal $\mathrm{D}_{\text {surf }}$, three boluses of different thicknesses $(0.5 \mathrm{~cm}, 1.0 \mathrm{~cm}$, and $1.5 \mathrm{~cm})$ were used with zero air gap from surface of the solid water phantom. Dose measurements indicated that 1.0 $\mathrm{cm}$ bolus was the most effective in providing a consistent high dose on the surface for 6 and 10 MV X-ray beams. Although $\mathrm{D}_{\text {Surf }}$ with $1.0 \mathrm{~cm}$ and $1.5 \mathrm{~cm}$ boluses were nearly identical, the $1.0 \mathrm{~cm}$ bolus is more efficient due to lesser attenuation. Figures 3(a)-(d) show measured $D_{\text {Surf }}$ and $d_{\max }$ respectively in solid water phantom for field sizes of $5 \times 5 \mathrm{~cm}^{2}, 10 \times 10 \mathrm{~cm}^{2}, 15 \times 15 \mathrm{~cm}^{2}$ and $20 \times 20$ $\mathrm{cm}^{2}$, with BSDs of $0 \mathrm{~cm}, 0.5 \mathrm{~cm}, 1.0 \mathrm{~cm}, 2.0 \mathrm{~cm}, 3.0 \mathrm{~cm}$, $4.0 \mathrm{~cm}, 5.0 \mathrm{~cm}$, and also for corresponding unbolused (open) beams.

Each curve is normalized to $100 \%$ at the reference point on the surface of the solid water phantom with 0 $\mathrm{cm}$ BSD. An increase in surface dose is shown in these figures when bolus is used. There is a negligible effect on the $\mathrm{D}_{\text {Surf }}$ with increasing the air gap for larger field sizes compared to small fields. For a field size $5 \times 5 \mathrm{~cm}^{2} \mathrm{D}_{\text {Surf }}$ decreases by $34 \%$ and $30 \%$ with a $5 \mathrm{~cm}$ air gap for 6 and $10 \mathrm{MV}$ photon beams respectively. A change in the $\mathrm{d}_{\max }$ is also observed with changing the air gap.

The $\mathrm{d}_{\max }$ measured for open $6 \mathrm{MV}$ and $10 \mathrm{MV}$ photon beams are $1.4 \mathrm{~cm}$ and $2.2 \mathrm{~cm}$ respectively. For smaller field sizes such as $5 \times 5 \mathrm{~cm}^{2}$ the relative $D_{\text {Surf }}$ is significantly affected by air gaps greater than $0.5 \mathrm{~cm}$. For larger field sizes such as $10 \times 10 \mathrm{~cm}^{2}$ and higher, relative $D_{\text {Surf }}$ is nearly the same for air gaps of $0 \mathrm{~cm}-5.0 \mathrm{~cm}$. For small field and $6 \mathrm{MV} \mathrm{d}_{\max }$ increases with increase in air gaps. For the $10 \mathrm{MV}$ beam a similar trend like $6 \mathrm{MV}$ beam was observed for $15 \times 15 \mathrm{~cm}^{2}$ and $20 \times 20 \mathrm{~cm}^{2}$ field sizes, however for smaller fields $\left(5 \times 5 \mathrm{~cm}^{2}\right.$ and 10 $\times 10 \mathrm{~cm}^{2}$ ) with increasing air gaps, $\mathrm{d}_{\max }$ first decreases and then increases as shown in Figure 3(d).

Figures 4(a)-(d) show the Eclipse generated IMRT (head \& neck) and 3DCRT (4-fields-pelvis) treatment plans with bolus placement and resultant dose distributions. These plans with air gaps of $0 \mathrm{~cm}$ to $5.0 \mathrm{~cm}$ were delivered on a Varian Linac and measured with Gafchromic ${ }^{\circledR}$ EBT films. Measured $D_{\text {Surf }}$ in Rando ${ }^{\circledR}$ phantom for these plans using a $1 \mathrm{~cm}$ thick bolus is presented in Fig- ures 5(a) and (b). For both the IMRT and 3DCRT plans $\mathrm{D}_{\text {Surf }}$ reduces with increasing air gaps by about $20 \%$.

\section{Discussion}

A special consideration is needed when using a bolus for dose buildup with smaller field sizes. It has been reported by Kassaee et al. [13] that the spoiler should not be placed at a distance less than $6 \mathrm{~cm}$ from skin surface in order to avoid loss of skin sparing during TBI. It means that if the air gap is less than $6 \mathrm{~cm}$, the spoiler will act as a bolus and the skin will receive a higher dose. Gray et al. [12] reported that even when electronic equilibrium is established in the material positioned before the air gap, there is a secondary region of dose buildup beyond the air gap to establish the electronic equilibrium when the air gap is greater than $5 \mathrm{~cm}$. They concluded that the air gap greater than $5 \mathrm{~cm}$ should be avoided, because the accuracy of Eclipse ${ }^{\mathrm{TM}}$ dose calculation beyond the secondary buildup region is out by $\sim 2.5 \%$. Based on these findings, we selected our air gaps 0 to $5 \mathrm{~cm}$ for the current study. Because bolus is only effective within a limited range of BSDs, positioning the bolus requires care on the part of the radiation therapist for accurate dose delivery to the surface.

Figures 3(a) and (b) show that, for a $1 \mathrm{~cm}$ water equivalent bolus placed above the phantom, an increase in the air gap decreases the dose measured on the surface for small field sizes (i.e., $5 \times 5 \mathrm{~cm}^{2}$ and $10 \times 10 \mathrm{~cm}^{2}$ ). On the other hand for field sizes larger than $10 \times 10 \mathrm{~cm}^{2}$, the $\mathrm{D}_{\text {Surf }}$ is less affected by different air gaps. The reason for lower $\mathrm{D}_{\text {Surf }}$ for small field sizes are, less scatter contribution from collimator and water phantom. Reduction in the scatter from bolus with increasing air gap, reaching the surface also reduces $\mathrm{D}_{\text {Surf. This loss of scatter radia- }}$ tion is mainly caused by the lateral spread of the scattered radiation within the air gap and is directly proportional to the size of the air gap for small field sizes. Small fields are used clinically for some treatments such as breast boost, and anal verge. In these scenarios the bolus is placed almost in contact with the skin. For larger field size such as chest wall the effect of gaps on the $D_{\text {Surf }}$ is minimal as shown in Figure 2(b). In general, the contri-

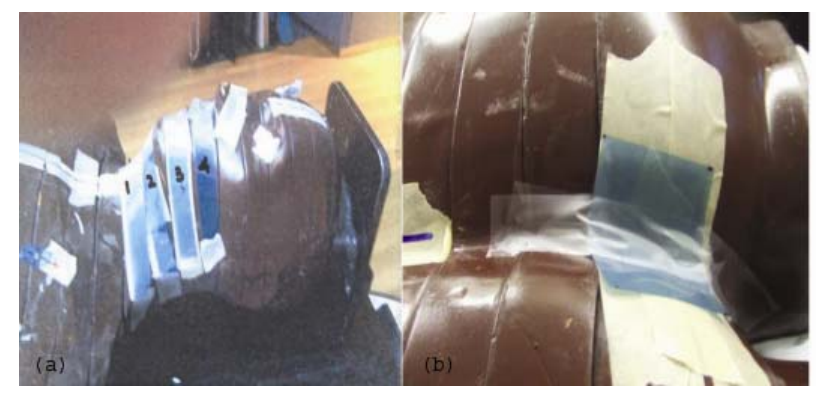

Figure 2. Geometrical setup for $D_{\text {Surf }}$ measurements on Rando ${ }^{\circledR}$ phantom. 

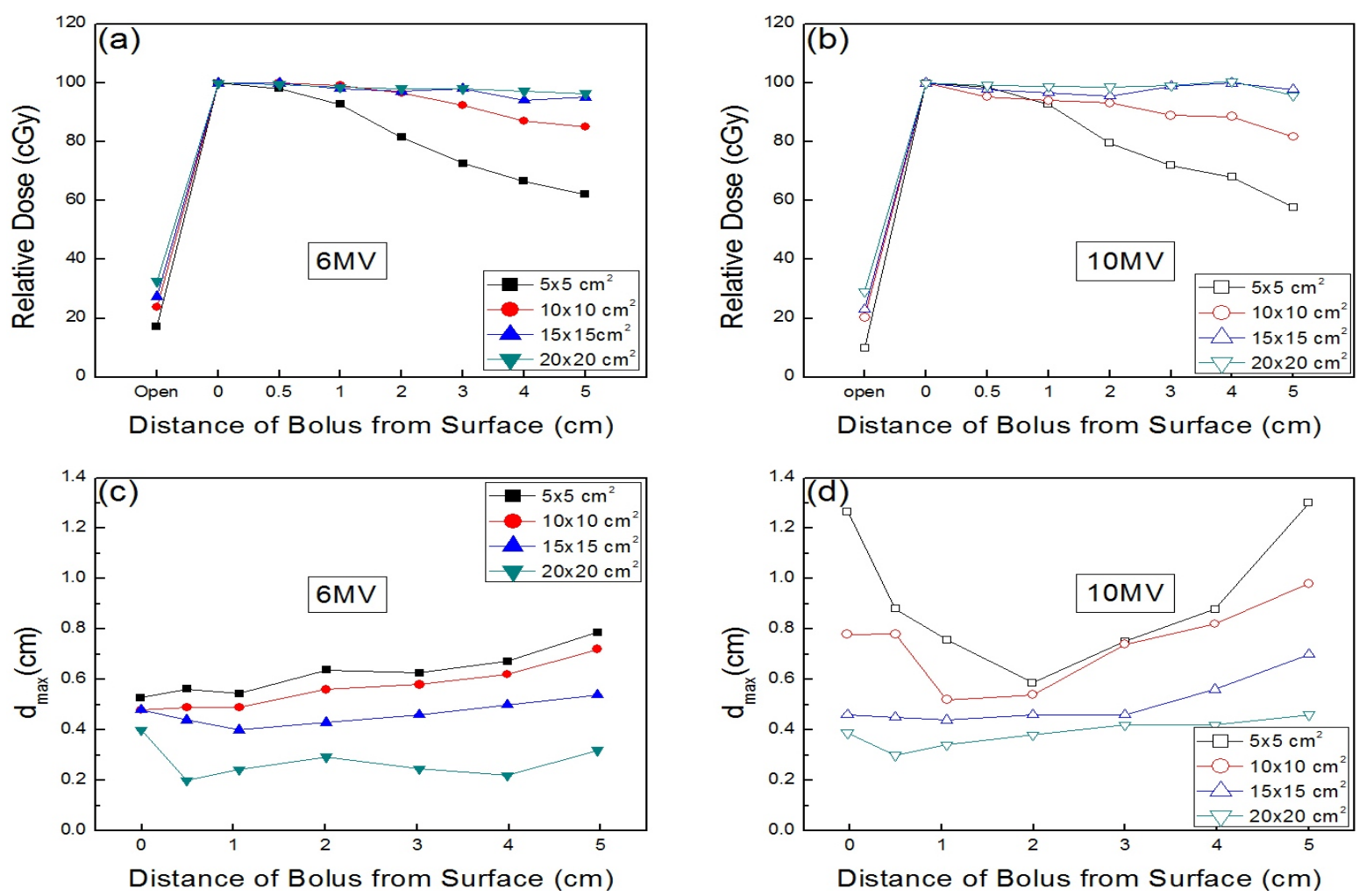

Figure 3. (a) and (b) Relative $D_{\text {Surf }}$ curve for 6 and $10 \mathrm{MV}$ beams $5 \times 5 \mathrm{~cm}^{2}, 10 \times 10 \mathrm{~cm}^{2}, 15 \times 15 \mathrm{~cm}^{2}$ and $20 \times 20 \mathrm{~cm}^{2}$ for 6 MV and 10 MV X-ray beams respectively, (c) and (d) Relation between $d_{\max }$ and BSD for field size $5 \times 5 \mathrm{~cm}^{2}, 10 \times 10 \mathrm{~cm}^{2}, 15$ $\times 15 \mathrm{~cm}^{2}$ and $20 \times 20 \mathrm{~cm}^{2}$ for $6 \mathrm{MV}$ and $10 \mathrm{MV}$ X-ray beams respectively.

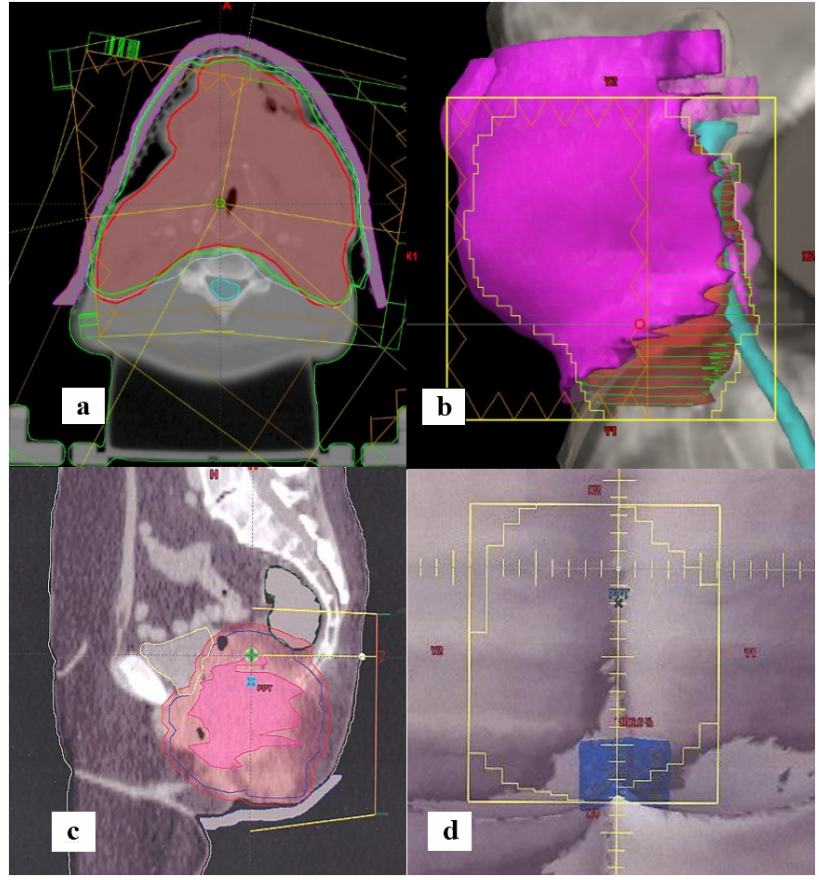

Figure 4. (a), (b), (c) and (d), Head \& Neck IMRT and Pelvis 3DCRT location of bolus and skin rendering.

bution of electrons generated within the bolus increases as the bolus gets closer to the phantom surface and as the field size increases.

The findings shown in Figures 3(a)-(d) indicate that both, field size and beam energy influence the dose buildup and $\mathrm{d}_{\max }$. For $6 \mathrm{MV}$ photon beam $\mathrm{d}_{\max }$ is less affected for all field sizes while for $10 \mathrm{MV}$ photon beam the relationship was only consistent for $15 \times 15 \mathrm{~cm}^{2}$ and $20 \times 20 \mathrm{~cm}^{2}$ filed sizes. For smaller field sizes the electronic equilibrium was established at greater depth than for larger field sizes as shown in Figures 3(c) and (d). The $d_{\max }$ is shifted deeper in water phantom for $10 \mathrm{MV}$ beam compared to $6 \mathrm{MV}$, as expected. The reason being the range of secondary electrons in the air is larger for 10 $\mathrm{MV}$ than for $6 \mathrm{MV}$ beam. Electrons from the bolus have limited range and affect the dose only close to the surface and up to $\mathrm{d}_{\max }$. In general $\mathrm{D}_{\text {Surf }}$ due to contamination electrons emanating from the bolus depends on the photon beam energy, air gaps, field size and thickness of the bolus.

The measured dose for IMRT and 3-DCRT treatments show similar effects to those observed with solid water phantom as shown in Figures 5(a) and (b). The reason for higher dose to skin for IMRT plan is mainly due to the presence of larger penumbra and overlapping fields.

\section{Conclusion}

The dose to the phantom surface in the presence of air 

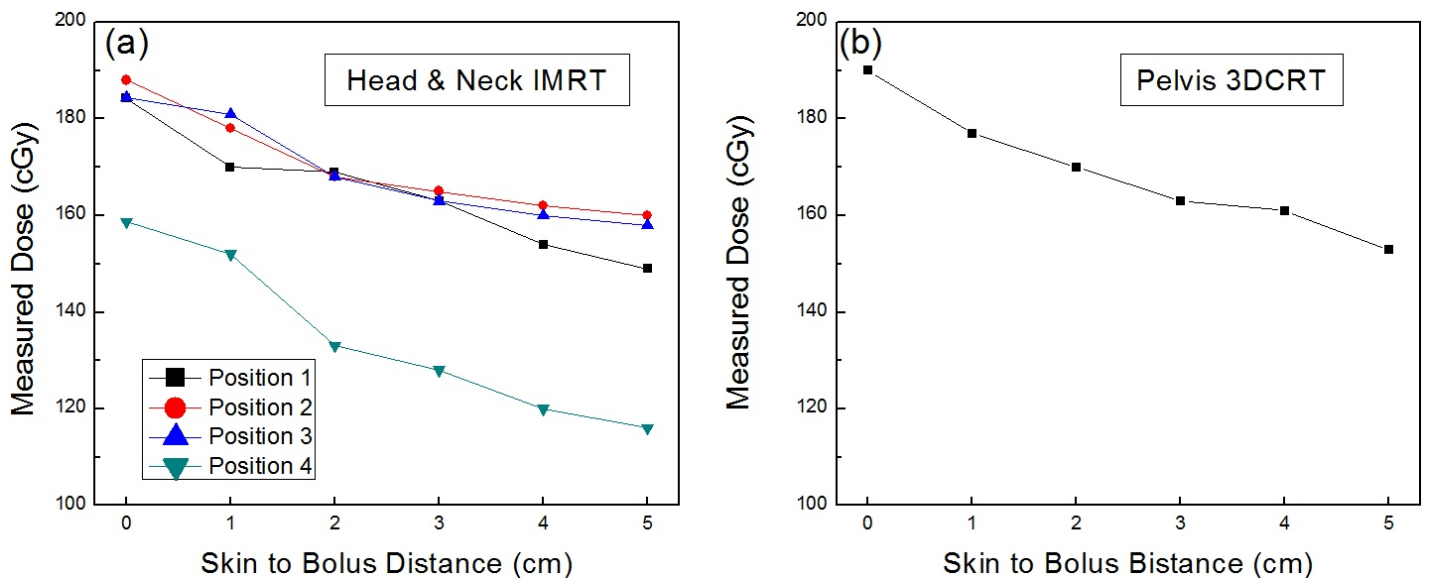

Figure 5. (a) Dose build up characteristics on skin surface for an IMRT 6 MV X-ray beam with 1cm of bolus material at 0 $5.0 \mathrm{~cm}$ air gaps; (b) Reduction in dose caused by varying air gaps under $1 \mathrm{~cm}$ of bolus for a 4-fields box 3DCRT plan.

gaps with bolus is less affected for large field sizes such as $15 \times 15 \mathrm{~cm}^{2}$ and greater. For larger field sizes $D_{\text {Surf }}$ greater than $95 \%$ was observed for larger air gapes of 5 $\mathrm{cm}$ as well. For IMRT and 3DCRT plans delivered to Rando $^{\circledR}, 94 \% D_{\text {Surf }}$ was observed for $1 \mathrm{~cm}$ air gap. Based on our results, special consideration is required when field sizes are smaller and surface contour variations are greater or when the bolus cover small area and at the border of the field. In general it is observed that the closer the bolus to the phantom surface is, the shallower the $\mathrm{d}_{\max }$ is for both $6 \mathrm{MV}$ and $10 \mathrm{MV}$ photon beams and all fields sizes. For both energies $d_{\max }$ is approximately proportional to air gaps.

\section{REFERENCES}

[1] F. M. Khan, “The Physics of Radiation Therapy," Lippincott Williams \& Wilkins, Philadelphia, 2010, p. 144.

[2] M. Stroka, J. Reguła and W. Łobodziec, "The Influence of the Bolus-Surface Distance on the Dose Distribution in the Build-Up Region," Reports of Practical Oncology \& Radiotherapy, Vol. 15, No. 6, 2010, pp. 161-164. doi:10.1016/j.rpor.2010.09.003

[3] K. I. Aneta, Ł. Włodzimierz, D. Marcin, N. Dorota and I. Tomasz, "Dose Distribution Homogeneity in Two TBI Techniques-Analysis of 208 Irradiated Patients Conducted in Stanislaw Leszczynski Memorial Hospital, Katowice," Reports of Practical Oncology \& Radiotherapy, Vol. 17, No. 6, 2012, pp. 367-375. doi:10.1016/j.rpor.2012.07.013

[4] J. B. Martin, C. Tsang and Y. Peter, "Effects on Skin Dose from Unwanted Air Gaps under Bolus in Photon Beam Radiotherapy," Radiation Measurements, Vol. 32, No. 3, 2000, pp. 201-204. doi:10.1016/S1350-4487(99)00276-0

[5] S. H. Hsu, R. Kulasekere and P. L. Roberson, "Analysis of Variation in Calibration Curves for Kodak XV Radiographic Film Using Model-Based Parameters," Journal of Applied Clinical Medical Physics, Vol. 11, No. 4, 2010, pp. 222-237.

[6] K. Alireza, B. Peter, Y. Ellen, et al., "Beam Spoilers versus Bolus for $6 \mathrm{mv}$ Photon Treatment of Head and Neck Cancers," Medical Dosimetry, Vol. 25, No. 3, 2000, pp. 127-131. doi:10.1016/S0958-3947(00)00038-8

[7] S. H. Hsu, P. L. Roberson, Y. Chen, et al., "Assessment of Skin Dose for Breast Chest Wall Radiotherapy as a Function of Bolus Material," Physics in Medicine \& Biology, Vol. 53, No. 10, 2008, pp. 2593-2606. doi:10.1088/0031-9155/53/10/010

[8] N. Lee, C. Chuang, J. M. Quivey, et al., "Skin Toxicity Due to IMRT for Head-and-Neck Carcinoma," International Journal of Radiation Oncology Biology Physics, Vol. 53, No. 3, 2002, pp. 630-637. doi:10.1016/S0360-3016(02)02756-6

[9] P. D. Higgins, E. H. Han, J. L. Yuan and C. K. Lee, "Evaluation of Surface and Superficial Dose for Head and Neck Treatments Using Conventional or IMRT Techniques," Physics in Medicine \& Biology, Vol. 52, 2007, pp. 1135-46. doi:10.1088/0031-9155/52/4/018

[10] N. Dogan and Glasgow, "Surface and Build-Up Region Dosimetry for Obliquely Incident IMRT 6MV X Rays," Medical Physics, Vol. 30, No. 12, pp. 3091-3096. doi:10.1118/1.1625116

[11] S. Yokoyama, P. L. Roberson, D. W. Litzenberg, et al., "Surface Buildup Dose Dependence on Photon Field Delivery Technique for IMRT," Journal of Applied Clinical Medical Physics, Vol. 5, No. 2, 2004, pp. 71-81. doi:10.1120/jacmp.2020.21706

[12] A. Gray, L. D. Oliver and P. N. Johnston, "The Accuracy of the Pencil Beam Convolution and Anistropic Analytical Algorithms in Predicting the Dose Effects Due to Attenuation from Immobilization Devices and Large Air Gaps," Medical Physics, Vol. 36, No. 7, 2009, pp. 31813191. doi:10.1118/1.3147204

[13] A. Kassaee, Y. Xiao, P. Bloch, et al., "Doses near the Surface during Total-Body Irradiation with 15 MV XRays," International Journal of Cancer, Vol. 96, 2001, pp. 125-130. doi:10.1002/ijc.10349 